\title{
Functions represented into fractional Taylor series
}

\author{
Ghiocel Groza ${ }^{1, *}$ and Marilena Jianu ${ }^{1, * *}$ \\ ${ }^{1}$ Technical University of Civil Eng. of Bucharest, Department of Mathematics and Computer Science, \\ 020396, Romania
}

\begin{abstract}
Fractional Taylor series are studied. Then solutions of fractional linear ordinary differential equations (FODE), with respect to Caputo derivative, are approximated by fractional Taylor series. The Cauchy-Kowalevski theorem is proved to show the existence and uniqueness of local solutions for FODE with Cauchy initial data. Sufficient conditions for the global existence of the solution and the estimate of error are given for the method using fractional Taylor series. Two illustrative numerical examples are given to demonstrate the validity and applicability of this method.
\end{abstract}

\section{Introduction}

Taylor series method is a useful tool to approximate solutions of the ordinary differential equations (ODE) (see, for example, [1], [9], [12] and references therein) or solutions of the partial differential equations (PDE) (see, for example, [2], [4]). One advantage of the analytic methods is that the accuracy of solution can be evaluated directly. Thus the approximate solution can be replaced into the equation and the initial or boundary conditions.

Fractional differential equations are useful tools for modeling many phenomena in fields of science and engineering (see [3], [5], [8], [11]). Several methods for approximation of solutions of ordinary differential equations were extended to fractional ordinary differential equations (FODE) (see, for example, [7]).

Fractional Taylor series method to approximate solutions for FODE, based on the corresponding Taylor's formula (see [10], [13]), can be found in [6], and references therein.

In this paper some properties of fractional Taylor series are given (see Theorems 1 and 2 ). We prove Cauchy-Kowalevski theorem in the case of linear FODE guaranteeing existence and uniqueness of local solutions with Cauchy initial data. Moreover, sufficient conditions for the existence of the global solution are given (see Theorem 3). By using the proof of the theorem we estimate the error in the method based on fractional Taylor series (see Remark 1). Finally, two numerical examples are presented to illustrate the results obtained.

\section{Fractional Taylor series}

Definition 1 A function $f:(0, \infty) \rightarrow \mathbb{R}$ is said to be of class $C_{\mu}(\mu \in \mathbb{R})$ if there exists $p>\mu$ such that $f(t)=t^{p} g(t), \forall t>0$, where $g:[0, \infty) \rightarrow \mathbb{R}$ is a continuous function. The function $f$ is said to be of class $C_{\mu}^{(n)}(n \in \mathbb{N})$ if $f^{(n)} \in C_{\mu}$.

\footnotetext{
*e-mail: grozagc@yahoo.com

**e-mail: marilena_jianu@yahoo.com
} 
Definition 2 Let $n=\lceil\alpha\rceil$ (where $\lceil x\rceil=\min \{z \in \mathbb{Z}: z \geq x\}$ denotes the ceiling function). The Caputo fractional derivative of order $\alpha \geq 0$ of a function $y \in C_{-1}^{(n)}$ is defined as

$$
D^{\alpha} y(t)= \begin{cases}\frac{1}{\Gamma(n-\alpha)} \int_{0}^{t} \frac{y^{(n)}(s)}{(t-s)^{1+\alpha-n}} d s, & \alpha \notin \mathbb{N} \\ y^{(\alpha)}(t), & \alpha \in \mathbb{N} .\end{cases}
$$

The Caputo fractional derivative has the following properties:

$$
\begin{gathered}
D^{\alpha}\left(\lambda_{1} y_{1}(t)+\lambda_{2} y_{2}(t)\right)=\lambda_{1} D^{\alpha} y_{1}(t)+\lambda_{2} D^{\alpha} y_{2}(t), \forall \lambda_{1}, \lambda_{2} \text { constants, } \\
D^{\alpha} t^{\gamma}=\frac{\Gamma(\gamma+1)}{\Gamma(\gamma-\alpha+1)} t^{\gamma-\alpha}, \quad \text { if } \gamma \geq \alpha, \\
D^{\alpha} t^{\gamma}=0, \text { if } \gamma \in \mathbb{N}, \gamma<\alpha .
\end{gathered}
$$

A series of functions of the form $\sum_{n \geq 0} a_{n} t^{n \alpha}$ with $\alpha>0$ is said to be a fractional power series. Notice that it is sufficient to study the fractional power series with $\alpha \in(0,1]$ because any fractional power series with $\alpha>1$ can be considered as a fractional power series of the form $\sum_{n \geq 0} a_{n}^{\prime} t^{n \alpha^{\prime}}$ with $\alpha^{\prime}=\frac{\alpha}{\lceil\alpha\rceil} \in(0,1]$.

Theorem 1 Let $\sum_{n \geq 0} a_{n} t^{n \alpha}, \alpha \in(0,1]$, be a fractional power series, let $R \geq 0$ be the radius of convergence of the power series $\sum_{n \geq 0} a_{n} x^{n}$ and $r=\left\{\begin{array}{l}R^{\frac{1}{\alpha}}, \text { if } R<\infty \\ \infty, \text { if } R=\infty .\end{array}\right.$ Then i) If $R>0$, then the series $\sum_{n \geq 0} a_{n} t^{n \alpha}$ converges absolutely and uniformly on $[0, b]$, for all $b \in(0, r)$ and there exists a positive integer $N(b)$ such that

$$
\left|a_{n}\right| \leq b^{-n \alpha}, \text { for all } n \geq N(b)
$$

ii) if $f:[0, r) \rightarrow \mathbb{R}$ is the sum of the fractional power series, $f(t)=\sum_{n \geq 0} a_{n} t^{n \alpha}, \forall t \in[0, r)$, then $f$ is continuous and there exists the Caputo derivative, $D^{\alpha} f:[0, r) \rightarrow \mathbb{R}$. Moreover, the series of the Caputo derivatives, $\sum_{n \geq 0} a_{n} D^{\alpha}\left(t^{n \alpha}\right)=\sum_{n \geq 1} a_{n} \frac{\Gamma(n \alpha+1)}{\Gamma((n-1) \alpha+1)} t^{(n-1) \alpha}$ converges absolutely and uniformly on $[0, b], \forall b \in(0, r)$ and

$$
D^{\alpha} f(t)=\sum_{n \geq 0} a_{n} D^{\alpha}\left(t^{n \alpha}\right)=\sum_{n \geq 1} a_{n} \frac{\Gamma(n \alpha+1)}{\Gamma((n-1) \alpha+1)} t^{(n-1) \alpha}, \forall t \in[0, r) .
$$

Proof. i) Since, for every $t \in[0, b],\left|a_{n} t^{n \alpha}\right| \leq\left|a_{n}\right|\left(b^{\alpha}\right)^{n}$ and the series $\sum_{n \geq 0}\left|a_{n}\right|\left(b^{\alpha}\right)^{n}$ is convergent, it follows that the series $\sum_{n \geq 0} a_{n} t^{n \alpha}$ converges absolutely and uniformly on $[0, b]$. Because $R=\frac{1}{\lim \sup \left|a_{n}\right|^{1 / n}}$, we get (3).

ii) Let $g:[0, R) \rightarrow \mathbb{R}$ be the sum of the power series: $g(x)=\sum_{n \geq 0} a_{n} x^{n}$, so $g(x)$ is continuous. Since $f(t)=g\left(t^{\alpha}\right)$ and the functions $g(x)$ and $t^{\alpha}$ are continuous, it follows that $f(t)$ is continuous.

It is enough to consider $\alpha<1$. By Stirling formula, $\lim _{n \rightarrow \infty} \sqrt[n]{\frac{\Gamma(n \alpha+1)}{\Gamma((n-1) \alpha+1)}}=1$. Hence the radius of convergence of the power series $\sum_{n \geq 1} a_{n} \frac{\Gamma(n \alpha+1)}{\Gamma((n-1) \alpha+1)} x^{n-1}$ is $R=\frac{1}{\limsup _{n \rightarrow \infty} \sqrt[n]{\left|a_{n}\right|}}$. Thus, the fractional power series $\sum_{n \geq 1} a_{n} \frac{\Gamma(n \alpha+1)}{\Gamma((n-1) \alpha+1)} t^{(n-1) \alpha}$ is convergent on $[0, r)$. 
Let $t \in(0, r)$ and $\sigma, \tau$ such that $0<\sigma \leq \tau \leq t$. The series

$$
\sum_{n \geq 0} a_{n}\left(s^{n \alpha}\right)^{\prime}=\sum_{n \geq 1} a_{n} \cdot n \alpha s^{n \alpha-1}=\alpha s^{-1} \sum_{n \geq 1} n a_{n} s^{n \alpha}
$$

converges absolutely and uniformly on $[\sigma, \tau]$ and $f^{\prime}(s)=\sum_{n \geq 1} a_{n} \cdot n \alpha s^{n \alpha-1}, \forall s \in[\sigma, \tau]$. Since $\alpha \in(0,1)$, for $s \in[\sigma, \tau]$, we obtain:

$$
\frac{1}{\Gamma(1-\alpha)} \int_{\sigma}^{\tau}(t-s)^{-\alpha} f^{\prime}(s) d s=\sum_{n \geq 1} \frac{a_{n} n \alpha}{\Gamma(1-\alpha)} \int_{\sigma}^{\tau} s^{n \alpha-1}(t-s)^{-\alpha} d s .
$$

For a fixed $\sigma>0$, we denote by $\varphi_{n}(\tau)=\frac{a_{n} n \alpha}{\Gamma(1-\alpha)} \int_{\sigma}^{\tau} s^{n \alpha-1}(t-s)^{-\alpha} d s, \tau \in[\sigma, t]$, and by $F(\tau)$ the sum of the series $\sum_{n \geq 1} \varphi_{n}(\tau)$. Then $F(\tau)=\frac{1}{\Gamma(1-\alpha)} \int_{\sigma}^{\tau}(t-s)^{-\alpha} f^{\prime}(s) d s, \forall \tau \in[\sigma, t)$. Since, for any $\tau \in[\sigma, t]$, we have $\left|\varphi_{n}(\tau)\right| \leq \frac{\left|a_{n}\right| n \alpha}{\Gamma(1-\alpha)} \cdot \int_{0}^{t} s^{n \alpha-1}(t-s)^{-\alpha} d s$

$$
=\frac{\left|a_{n}\right| n \alpha}{\Gamma(1-\alpha)} t^{(n-1) \alpha} B(n \alpha, 1-\alpha)=\left|a_{n}\right| t^{(n-1) \alpha} \frac{\Gamma(n \alpha+1)}{\Gamma((n-1) \alpha+1)}
$$

and the series $\sum_{n \geq 1}\left|a_{n}\right| t^{(n-1) \alpha} \frac{\Gamma(n \alpha+1)}{\Gamma((n-1) \alpha+1)}$ is convergent, it follows that the series $\sum_{n \geq 1} \varphi_{n}(\tau)$ is absolutely uniformly convergent on $[\sigma, t]$. Thus its sum, $F(\tau)$, is continuous at $t$, and

$$
\frac{1}{\Gamma(1-\alpha)} \int_{\sigma}^{t}(t-s)^{-\alpha} f^{\prime}(s) d s=\sum_{n \geq 1} \frac{a_{n} n \alpha}{\Gamma(1-\alpha)} \int_{\sigma}^{t} s^{n \alpha-1}(t-s)^{-\alpha} d s .
$$

This equality holds for any $\sigma \in(0, t]$.

Similarly, it can be proved that the series of functions $\sum_{n \geq 1} \psi_{n}(\sigma)$, with $\psi_{n}(\sigma)=$ $\frac{a_{n} n \alpha}{\Gamma(1-\alpha)} \int_{\sigma}^{t} s^{n \alpha-1}(t-s)^{-\alpha} d s$ converges absolutely and uniformly on $[0, t]$. Hence its sum is continuous at 0 and it follows that

$$
\frac{1}{\Gamma(1-\alpha)} \int_{0}^{t}(t-s)^{-\alpha} f^{\prime}(s) d s=\sum_{n \geq 1} \frac{a_{n} n \alpha}{\Gamma(1-\alpha)} \int_{0}^{t} s^{n \alpha-1}(t-s)^{-\alpha} d s,
$$

which is equivalent to:

$$
D^{\alpha} f(t)=\sum_{n \geq 1} \frac{a_{n} n \alpha}{\Gamma(1-\alpha)} t^{(n-1) \alpha} B(n \alpha, 1-\alpha)=\sum_{n \geq 1} a_{n} \frac{\Gamma(n \alpha+1)}{\Gamma((n-1) \alpha+1)} t^{(n-1) \alpha} .
$$

A real function $f$ is said to be representable into an $\alpha$-fractional Taylor series on the interval $I=[0, b)$ if it can be written as the sum of a fractional power series:

$$
f(t)=\sum_{n \geq 0} a_{n} t^{n \alpha}, \forall t \in I .
$$

If $I=[0, b]$, then $f$ is said to be representable into an $\alpha$ - fractional Taylor series on $I$ if there exists $b^{\prime}>b$ and $f$ is representable into an $\alpha$ - fractional Taylor series on $\left[0, b^{\prime}\right)$.

For $I=[0, b)$ or $I=[0, b]$, denote by $\mathcal{T}_{\alpha}(I)$ the set of all functions $f: I \rightarrow \mathbb{R}$ which are representable into an $\alpha$ - fractional Taylor series on $I$.

For any $\alpha>0$ and $n \in \mathbb{N}$, we denote by $D^{n \alpha} f(t)=\underbrace{D^{\alpha} D^{\alpha} \ldots D^{\alpha}}_{n \text {-times }} f$. If $f \in \mathcal{T}_{\alpha}(I)$, by (1) and (2), it follows that $D^{n \alpha} f=D^{\alpha_{1}} f$, where $\alpha_{1}=n \alpha$. 
Corollary 1 If $f \in \mathcal{T}_{\alpha}(I)$ is represented in (5) then, for any $k \in \mathbb{N}$, there exists $D^{k \alpha} f \in C(I)$ and

$$
D^{k \alpha} f(t)=\sum_{n \geq k} a_{n} \frac{\Gamma(n \alpha+1)}{\Gamma((n-k) \alpha+1)} t^{(n-k) \alpha}, \forall t \in I .
$$

The coefficients of the $\alpha$ - fractional Taylor series (5) are given by: $a_{n}=\frac{D^{n \alpha} f(0)}{\Gamma(n \alpha+1)}$.

Theorem 2 Let $f:[0, b) \rightarrow \mathbb{R}$, where $b>1$, be a function such that there exists $D^{n \alpha} f(t) \in$ $C([0,1])$ for any $n \in \mathbb{N}, \alpha \in(0,1]$. We denote $M_{n}=\left\|D^{n \alpha} f(t)\right\|=\sup _{t \in[0,1]}\left|D^{n \alpha} f(t)\right|$. If $M_{n}=$ $o(\Gamma(\alpha n+1))$, then $f$ is representable into a fractional Taylor series on $[0,1]$ and

$$
f(t)=\sum_{n=0}^{\infty} \frac{D^{n \alpha} f(0)}{\Gamma(n \alpha+1)} t^{n \alpha}, \quad t \in[0,1]
$$

Proof. By Taylor's Formula (see [10]) we have that

$$
\left|f(t)-\sum_{k=0}^{n} \frac{D^{k \alpha} f(0)}{\Gamma(k \alpha+1)} t^{k \alpha}\right|=\left|\frac{D^{(n+1) \alpha} f(\tau)}{\Gamma((n+1) \alpha+1)} t^{(n+1) \alpha}\right|, \tau \in[0, t] .
$$

Hence, it follows (7).

\section{Fractional differential equations}

Consider $\alpha \in(0,1]$ and the linear fractional differential equation

$$
D^{n \alpha} y(t)+a_{1}(t) D^{(n-1) \alpha} y(t)+\ldots+a_{n-1}(t) D^{\alpha} y(t)+a_{n}(t) y(t)=f(t), t \in[0, b] .
$$

Lemma 1 Suppose that $b^{\prime}>b, f \in \mathcal{T}_{\alpha}\left(\left[0, b^{\prime}\right)\right), a_{j} \in \mathcal{T}_{\alpha}\left(\left[0, b^{\prime}\right)\right), j=1,2, \ldots, n$,

$$
f(t)=\sum_{k=0}^{\infty} b_{k} t^{k \alpha} \text { and } a_{j}(t)=\sum_{r=0}^{\infty} d_{j r} t^{\alpha \alpha}, j=1,2, \ldots, n .
$$

If $c_{0}, c_{1}, \ldots, c_{n-1}$ are arbitrary real numbers and, for all $k \geq 0$,

$$
c_{n+k}=\frac{\Gamma(k \alpha+1)}{\Gamma((k+n) \alpha+1)}\left(b_{k}-\sum_{j=1}^{n} \sum_{r=0}^{k} \frac{\Gamma((k+n-r-j) \alpha+1)}{\Gamma((k-r) \alpha+1)} d_{j r} c_{k+n-r-j}\right),
$$

then

$$
y(t)=\sum_{k=0}^{\infty} c_{k} t^{k \alpha} \in \mathcal{T}_{\alpha}([0, b])
$$

and $y(t)$ is a solution of the equation (8).

Proof. Consider $\delta, \delta_{1} \in\left(b, b^{\prime}\right)$ where $\delta<\delta_{1}$. Then by Theorem 1, there exists a positive integer $s_{1}=s_{1}(\delta) \geq n$ such that, for all $s \geq s_{1}$,

$$
\left|b_{s}\right| \leq \frac{1}{\delta^{\alpha s}}, \quad\left|d_{j s}\right| \leq \frac{1}{\delta_{1}^{\alpha S}}, j=1,2, \ldots, n .
$$

Hence we may choose a constant $\Delta \geq 1$ such that, for every $s$,

$$
\left|d_{j s}\right| \leq \frac{\Delta}{\delta_{1}^{\alpha S}}, j=1,2, \ldots, n .
$$


We denote

$$
h_{j}(s)=\frac{\Gamma((s-j) \alpha+1)}{\Gamma(s \alpha+1)}, j=1,2, \ldots, n, \quad g(s):=\sum_{j=1}^{n} \delta^{\alpha j} h_{j}(s+1-n)
$$

and $S_{1}(s):=\sum_{r=0}^{n_{1}}\left(\frac{\delta}{\delta_{1}}\right)^{\alpha r} g(s-r)$, where $n_{1}=n_{1}(s)=s+1-2 n$.

Since $h_{j}(s)=\frac{1}{\Gamma(\alpha j)} B((s-j) \alpha+1, \alpha j)$, it follows that for all $s \geq n$,

$$
h_{j}(s) \geq h_{j}(s+1), \forall j=\overline{1, n} .
$$

Hence, $g(s)$ is a decreasing function for $s \geq 2 n-1$ and it follows that, for any $s \geq 2 n-1$, $\{g(s-r)\}_{r=\overline{0, n_{1}}}$ is an increasing sequence. Since $\left\{\left(\frac{\delta}{\delta_{1}}\right)^{\alpha r}\right\}_{r=\overline{0, n_{1}}}$ is a decreasing sequence, by Chebyshev's sum inequality we have

$$
\frac{1}{n_{1}+1} \sum_{r=0}^{n_{1}}\left(\frac{\delta}{\delta_{1}}\right)^{\alpha r} g(s-r) \leq\left(\frac{1}{n_{1}+1} \sum_{r=0}^{n_{1}}\left(\frac{\delta}{\delta_{1}}\right)^{\alpha r}\right) \cdot\left(\frac{1}{n_{1}+1} \sum_{r=0}^{n_{1}} g(s-r)\right)
$$

and it follows that

$$
S_{1}(s) \leq \frac{1}{1-\left(\frac{\delta}{\delta_{1}}\right)^{\alpha}} \cdot \frac{1}{n_{1}+1} \sum_{p=2 n-1}^{s} g(p)
$$

By Stirling's formula, we get $\lim _{s \rightarrow \infty} g(s)=0$, hence $\lim _{s \rightarrow \infty} \frac{1}{n_{1}+1} \sum_{p=2 n-1}^{s} g(p)=0$ and consequently, $\lim _{s \rightarrow \infty} S_{1}(s)=0$. Consider $\varepsilon_{i} \in(0,1), i=1,2,3$, such that

$$
\varepsilon_{1}+\varepsilon_{2}+\varepsilon_{3} \leq 1
$$

We can choose a positive integer $s_{2}=s_{2}(\delta) \geq s_{1}, s_{2} \geq 2 n-1$ such that, for all $s \geq s_{2}$,

$$
S_{1}(s) \leq \frac{\varepsilon_{1}}{\Delta} .
$$

We denote by $S_{2}(s):=\sum_{r=n_{1}+1}^{s+1-n}\left(\frac{\delta}{\delta_{1}}\right)^{\alpha r} \sum_{j=1}^{n} \delta^{\alpha j} \frac{\Gamma((s+1-n-r-j) \alpha+1)}{\Gamma((s+1-n-r) \alpha+1)}$, where $\Gamma(m)=1$ if $m \leq 0$ and let $\gamma=\min _{k=\overline{0, n-1}} \Gamma(k \alpha+1), \Gamma=\max _{k=\overline{0, n-2}} \Gamma(k \alpha+1)$. Then: $S_{2}(s) \leq n\left(\frac{\delta}{\delta_{1}}\right)^{\alpha n_{1}} \frac{\Gamma}{\gamma} \sum_{j=1}^{n} \delta^{\alpha j}$ and it follows that $\lim _{s \rightarrow \infty} S_{2}(s)=0$. Hence, there exists a positive integer $s_{3}=s_{3}(\delta) \geq s_{2}$ such that, for all $s \geq s_{3}$,

$$
S_{2}(s) \leq \frac{\varepsilon_{2}}{\Delta}
$$

We consider $C_{1} \geq \frac{1}{\varepsilon_{3}}$ such that, for all $s \leq s_{3}$,

$$
\left|c_{s}\right| \leq C_{1} \frac{\Gamma((s-n) \alpha+1)}{\Gamma(s \alpha+1) \delta^{\alpha(s-n)}} .
$$

This begins an inductive proof. For $s \geq s_{3}$, assume (17) true for all $s^{\prime} \leq s$ and we'll prove that

$$
\left|c_{s+1}\right| \leq C_{1} \frac{\Gamma((s+1-n) \alpha+1)}{\Gamma((s+1) \alpha+1) \delta^{\alpha(s+1-n)}} .
$$


By (9), (11), (12) and (17) we get

$$
\begin{aligned}
\left|c_{s+1}\right| \leq & \frac{\Gamma((s+1-n) \alpha+1)}{\Gamma((s+1) \alpha+1) \delta^{\alpha(s+1-n)}}\left(1+\sum_{j=1}^{n} \sum_{r=0}^{s+1-n} \frac{\Delta \Gamma((s+1-r-j) \alpha+1)}{\Gamma((s+1-n-r) \alpha+1) \delta_{1}^{\alpha r}}\right. \\
\cdot & \left.\frac{C_{1} \Gamma((s+1-n-r-j) \alpha+1)}{\Gamma((s+1-r-j) \alpha+1) \delta^{-\alpha(r+j)}}\right)=C_{1} \frac{\Gamma((s+1-n) \alpha+1)}{\Gamma((s+1) \alpha+1) \delta^{\alpha(s+1-n)}} \\
& \cdot\left(\frac{1}{C_{1}}+\Delta \sum_{r=0}^{s+1-n}\left(\frac{\delta}{\delta_{1}}\right)^{\alpha r} \sum_{j=1}^{n} \delta^{\alpha j} \frac{\Gamma((s+1-n-r-j) \alpha+1)}{\Gamma((s+1-n-r) \alpha+1)}\right) \\
& \leq C_{1} \frac{\Gamma((s+1-n) \alpha+1)}{\Gamma((s+1) \alpha+1) \delta^{\alpha(s+1-n)}}\left(\frac{1}{C_{1}}+\Delta\left(S_{1}(s)+S_{2}(s)\right)\right) .
\end{aligned}
$$

Thus, by (14), (15) and (16), we get (18), and (17) holds for every $s$.

Now, by (17), it follows that $\limsup \left|c_{s}\right|^{1 / s} \leq \frac{1}{\delta^{\alpha}}$. Since this is true for all $\delta \in$ $\left(b, b^{\prime}\right)$ it follows that (10) holds. Finally, by Theorem 1 and (9), we get the lemma.

The following result is an extension of the Cauchy-Kowalevski theorem in the case of linear fractional ordinary differential equation.

Theorem 3 Suppose that $b^{\prime}>b, f, a_{j} \in \mathcal{T}_{\alpha}\left(\left[0, b^{\prime}\right)\right), j=1,2, \ldots, n$ and $y_{i}^{(0)}, i=0,1, \ldots, n-1$, arbitrary real numbers. Then there exists a solution $y=y(t) \in \mathcal{T}_{\alpha}([0, b])$ of the equation (8), uniquely determined such that

$$
D^{i \alpha} y(0)=y_{i}^{(0)}, i=0,1, \ldots, n-1 .
$$

Proof. By Lemma 1 the equation (8) has a solution of the form (10), where $c_{i}, i=0,1, \ldots, n-1$ are arbitrary real numbers. If $c_{i}=\frac{y_{i}^{(0)}}{\Gamma(i \alpha+1)}$, by Corollary 1 , we get a solution in $\mathcal{T}_{\alpha}([0, b]$ of the initial problem (8), (19). Since every solution from $\mathcal{T}_{\alpha}([0, b]$ of $(8)$ has the form given in Lemma 1 it follows the theorem.

Remark 1 Under the hypotheses of Theorem 3 consider the solution $y=y(t)$ of the equation (8), $t \in[0, b]$, which verifies the Cauchy initial data (19). For a fixed $m$, by (9), we can find $c_{s}$, for all $s \leq m$. As in the proof of Lemma 1 , we get $s_{i}, i=1, \ldots, 3$ and a constant $C_{1}$ such that (17) holds. Then for any $m \geq s_{3}$, we approximate the solution by a fractional polynomial

$$
P_{m}(t)=\sum_{k=0}^{m} c_{k} t^{\alpha k}
$$

Thus $y(t)=P_{m}(t)+R_{m}(t)$ and $E_{m}=\sup _{t \in[0, b]}\left|R_{m}(t)\right|$ is the error of the approximation. By (17), for $m \geq 1 / \alpha+n, \delta \in\left(b, b^{\prime}\right)$, it follows that

$$
\left|R_{m}(t)\right| \leq \sum_{k=m+1}^{\infty}\left|c_{k} t^{\alpha k}\right| \leq C_{1} \sum_{k=m+1}^{\infty} \frac{\Gamma((k-n) \alpha+1) b^{\alpha k}}{\Gamma(k \alpha+1) \delta^{\alpha(k-n)}} \leq C_{1} \int_{m}^{\infty} \frac{\Gamma((s-n) \alpha+1) b^{\alpha s} d s}{\Gamma(s \alpha+1) \delta^{\alpha(s-n)}} .
$$

Hence we get

$$
E_{m} \leq C_{1} \int_{m}^{\infty} \frac{\Gamma((s-n) \alpha+1) b^{\alpha s} d s}{\Gamma(s \alpha+1) \delta^{\alpha(s-n)}} .
$$




\section{Numerical examples}

Example 1 Consider the Bagley-Torvik equation

$$
D^{2} y(t)+A D^{3 / 2} y(t)+B y(t)=f(t)=\sum_{k=0}^{\infty} \frac{t^{k / 2}}{\Gamma(k / 2+1)}, A, B \in \mathbb{R}, t \in(0,1),
$$

with the initial conditions

$$
D^{i / 2} y(0)=y_{0}^{(i)}, i=0,1,2,3 .
$$

By Theorem 3, where $\alpha=1 / 2$, the initial value problem (22), (23) has a uniquely determined solution of the form (10), where $c_{k}=\frac{y_{0}^{(k)}}{\Gamma(k \alpha+1)}, k=0,1,2,3$, and, from (9), for $k \geq 4, c_{k}$ are obtained in the following way

$$
c_{k}=\frac{1}{\Gamma(k / 2+1)}-A \frac{\Gamma((k-1) / 2+1)}{\Gamma(k / 2+1)} c_{k-1}-B \frac{\Gamma((k-4) / 2+1)}{\Gamma(k / 2+1)} c_{k-4}
$$

We approximate the solution by a fractional polynomial given in (20).

Consider, for example, $A=2, B=1, y_{0}^{(0)}=1, y_{0}^{(1)}=-\Gamma(3 / 2), y_{0}^{(2)}=2 \Gamma(2)$ and $y_{0}^{(3)}=\Gamma(5 / 2)$. Since $f(t)$ is the Mittag-Leffler function which converges for all $t \in \mathbb{R}$ and $a_{j}(t)$ are constant functions we can chose any $b^{\prime}, \delta$ and $\delta_{1}$ large enough. We take, for example $\delta=1.2$ and $\delta_{1}=10^{4}$. By using the notations from the proof of Lemma 1 we can choose $s_{1}=4$ and $\Delta=2, \varepsilon_{1}=0.98$ and $\varepsilon_{2}=\varepsilon_{3}=10^{-2}$. We can take $s_{2}=s_{3}=80$ and, by (17), we may choose $C_{1}=100$. For $m=100$, Table 1 shows the approximate solution for the problem. By (21) we obtain the estimation of the error $E_{100} \leq 100 \int_{100}^{\infty} \frac{\Gamma((s-4) / 2+1) d s}{\Gamma(s / 2+1) \delta^{(s-4) / 2}} \leq 10^{-4}$.

Table 1. Approximate solutions for the problem (22),(23)

\begin{tabular}{cllllll}
\hline$t$ & 0.0 & 0.1 & 0.2 & 0.3 & 0.4 & 0.5 \\
\hline$P_{m}(t)$ & 1.000000 & .907030 & 1.013929 & 1.160238 & 1.329894 & 1.516710 \\
\hline \hline$t$ & 0.6 & 0.7 & 0.8 & 0.9 & 1.0 & \\
\hline$P_{m}(t)$ & 1.717670 & 1.931200 & 2.156529 & 2.393409 & 2.641977 & \\
\hline
\end{tabular}

Remark 2 Usually the Bagley-Torvik equation is presented in the case when $y \in C^{2}([0,1])$. Then $D^{2 \alpha}(t)=D y(t)=y^{\prime}(t), D^{4 \alpha}(t)=D^{2} y(t)=y^{\prime \prime}(t)$ and $c_{1}=c_{3}=0$. Consequently, the initial conditions $(23)$ becomes $y(0)=y_{0}^{(0)}$ and $y^{\prime}(0)=y_{0}^{(1)}$.

Example 2 Let us consider the inhomogeneous fractional Ayre equation (Ex. 7.10 from [5])

$$
D^{2 \alpha} y(t)-t^{\alpha} y(t)=1-t^{\alpha}+t^{2 \alpha}, t \in[0, b], \alpha \in(0,1), y(0)=0, D^{\alpha} y(0)=0 .
$$

In this case $n=2, a_{1}=0, a_{2}=-t^{\alpha}$ and by (9), for $k \geq 2$, we find

$$
c_{k}=\frac{\Gamma((k-2) \alpha+1)}{\Gamma(k \alpha+1)}\left(b_{k-2}+c_{k-3}\right), c_{-1}=0 .
$$

For $\alpha=0.9, b=1$, we approximate the solution by a fractional polynomial given in (20).

Consider $y_{0}^{(0)}=0, y_{0}^{(1)}=0, \delta=1.2, \delta_{1}=20$ and $\Delta=\delta_{1}^{\alpha}=14.82269$. Then we may choose $s_{1}=2, \varepsilon_{1}=0.98$ and $\varepsilon_{2}=\varepsilon_{3}=0.01$. We can take $s_{2}=s_{3}=40$ and finally, by (17), we may choose $C_{1}=100$. For $m=40$, Table 2 shows the approximate solution for the problem. In this case an estimation of the error is $E_{50} \leq 100 \int_{50}^{\infty} \frac{\Gamma(0.9(s-2)+1) d s}{\Gamma(0.9 s+1) \delta^{0.9(s-2)}} \leq 10^{-4}$. 
Table 2. Approximate solutions for the problem (25)

\begin{tabular}{cllllll}
\hline$t$ & 0.0 & 0.1 & 0.2 & 0.3 & 0.4 & 0.5 \\
\hline$P_{m}(t)$ & .000000 & .009026 & .030343 & .061202 & .100528 & .148011 \\
\hline \hline$t$ & 0.6 & 0.7 & 0.8 & 0.9 & 1.0 & \\
\hline$P_{m}(t)$ & .203891 & .268881 & .344141 & .431276 & .532358 & \\
\hline
\end{tabular}

\section{Conclusion}

In this paper an extension of the Cauchy-Kowalevski theorem for linear FODE and sufficient conditions for the global existence of the solution are presented. As a result, the solution of such an equation can be approximated by a fractional Taylor polynomial and an estimation of the error is given. The validity and applicability of the method is demonstrated by illustrative examples.

\section{References}

[1] R. Barrio, M. Rodriguez, A. Abad, F. Blesa, Breaking the limits: The Taylor series method, Appl. Math. Comput. 217, 7940-7954 (2011)

[2] B. Bülbül, M. Sezer, A Taylor matrix method for the solution of a two-dimensional linear hyperbolic equation, Appl. Math. Lett., 24(10), 1716-1720 (2011)

[3] K. Diethelm, The analysis of fractional differential equations. An application-oriented exposition using differential operators of Caputo type (Springer, Berlin, 2010)

[4] G. Groza, M. Razzaghi, A Taylor series method for the solution of the linear initialboundary-value problems for partial differential equations, Comput. Math. Appl., 66(7), 1329-1343 (2013)

[5] A. A. Kilbas, H. M. Srivastava, J. J. Trujillo, Theory and applications of fractional differential equations (Elsevier, Amsterdam, 2006)

[6] V. S. Krishnasamy, S. Mashayekhi, M. Razzaghi, Numerical solutions of fractional differential equations by using fractional Taylor basis, IEEE/CAA Journal of Automatica Sinica, 4(1), 98-106 (2017)

[7] C. Li, F. Zeng, Numerical methods for fractional calculus (CRC Press, Taylor\&Francis Group, Boca Raton, 2015)

[8] K. S. Miller, B. Ross, An introduction to the fractional calculus and fractional differential equations (John Willey\&Sons, New York, 1993)

[9] R. D. Neidinger, Directions for computing truncated multivariate Taylor series, Math. of Comput. 74(249), 321-340 (2004)

[10] Z. Odibat, N. Shawagfeh, Generalized Taylor's formula, Appl. Math. Comput. 186(1), 286-293 (2007)

[11] I. Podlubny, Fractional differential equations (Academic Press, New York, 1999)

[12] M. Razzaghi, M. Razzaghi, Solution of linear two-point boundary value problems with time-varying coefficients via Taylor series, Int. J. Syst. Sci. 20(11), 2075-2084 (1989)

[13] J. J. Trujillo, M. Rivero, B. Bonilla, On a Riemann-Liouville generalized Taylor's formula, J. Math. Anal. Appl. 231, 255-265 (1999) 